\title{
PRODUCTS OF HERMITIAN MATRICES AND SYMMETRIES
}

\section{HEYDAR RADJAVI}

It is the main purpose of this note to prove that every complex matrix with real determinant is the product of four hermitian matrices; Theorem 2 is an actually stronger result. Every real square matrix is the product of two real hermitian matrices [1]; this is a special case of our Theorem 1 which is of interest in itself, if it is indeed new. Theorem 3 was motivated by a theorem of Halmos and Kakutani [3] who proved that every unitary operator on an infinitedimensional Hilbert space is the product of four symmetries (i.e., operators that are hermitian and unitary). We also show that the number of factors in these results cannot be reduced in general.

TheOREM 1. Let $F$ be any field and let $A$ be a square matrix over $F$. Then $A$ is the product of two symmetric matrices over $F$.

Proof. If $S_{1}, S_{2}, T$ are matrices of the same size over $F$, if $S_{j}=S_{j}$, $j=1,2$, and if $T$ is invertible, then $T^{-1}\left(S_{1} S_{2}\right) T$ is the product of two symmetric matrices $T^{-1} S_{1}\left(T^{t}\right)^{-1}$ and $T^{t} S_{2} T$ over $F$. Hence we can assume, with no loss of generality, that $A$ is in the rational form [4], i.e., $A=\operatorname{diag}\left(A_{1}, \cdots, A_{m}\right)$, where each $A_{i}$ is of the form

$$
B=\left(\begin{array}{cccccc}
0 & 0 & 0 & \cdots & 0 & a_{1} \\
1 & 0 & 0 & \cdots & 0 & a_{2} \\
0 & 1 & 0 & \cdots & 0 & a_{3} \\
\vdots & & & & \\
0 & 0 & 0 & \cdots & 1 & a_{n}
\end{array}\right)
$$

Hence it suffices to prove the theorem for a matrix of the form $B$ : The matrix

$$
C=\left(\begin{array}{rrrrrrr}
a_{2} & a_{3} & a_{4} & \cdots & a_{n-1} & a_{n} & -1 \\
a_{3} & a_{4} & a_{5} & \cdots & a_{n} & -1 & 0 \\
: & & & & & & \\
a_{n} & -1 & 0 & \cdots & 0 & 0 & 0 \\
-1 & 0 & 0 & \cdots & 0 & 0 & 0
\end{array}\right)
$$

is symmetric and invertible over $F$. Computation shows that $B C$ is

Received by the editors June 24, 1968. 
also symmetric so that $B=(B C) C^{-1}$ is the product of two symmetric matrices over $F$.

Theorem 2. Let $A$ be a matrix over any subfield $F$ of complex numbers. Then $A$ is the product of four hermitian matrices over $F$ if and only if $\operatorname{det} A$ is real.

Proof. Let $\operatorname{det} A$ be real. The property of being the product of four hermitian matrices over $F$ is invariant under similarity over $F$ (by an argument analogous to the one given in the proof of Theorem 1). Hence we can assume that $A$ is in the rational form $\operatorname{diag}\left(A_{1}, \cdots, A_{m}\right)$, where

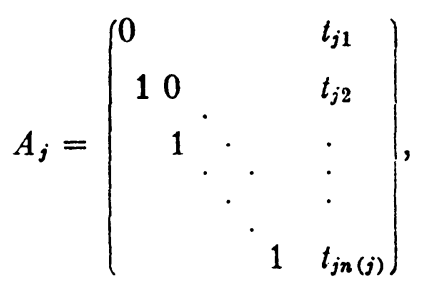

$j=1, \cdots, m$. Then $\operatorname{det} A= \pm t_{11} t_{21} \cdots t_{m 1}$; hence assume that if $\operatorname{det} A=0$, then $t_{m 1}=0$.

Let $n=n(1)+\cdots+n(m)$ and let $K$ denote the $n \times n$ matrix $\left(E_{2}, E_{3}, \cdots, E_{n}, E_{1}\right)$, where $E_{i}$ denotes the $i$ th column of the identity matrix. Then $A=B K$, where

$$
B=\left(\begin{array}{lllll}
B_{1} & C_{1} & 0 & \cdots & 0 \\
0 & B_{2} & C_{2} & \cdots & 0 \\
\cdot & & & & \\
\dot{0} & \cdots & B_{m-1} & C_{m-1} \\
C_{m} & \cdots & 0 & B_{m}
\end{array}\right)
$$

for each $j, B_{j}$ is an $n(j) \times n(j)$ block of the form $\operatorname{diag}(0,1,1, \cdots, 1)$, and

$$
C_{j}=\left(\begin{array}{llll}
t_{j 1} & 0 & \cdots & 0 \\
t_{j 2} & 0 & \cdots & 0 \\
\vdots & & & \\
t_{j n(j)} & 0 & \cdots & 0
\end{array}\right) .
$$

The matrix $K$ is the product of two hermitian matrices over $F$; in fact, by Theorem 1, it is the product of two symmetric matrices over the field of rational real numbers. We now proceed to prove that $B$ is also the product of two hermitian matrices over $F$. In view of 
Theorem 1 it suffices to show that $B$ is similar over $F$ to a real matrix.

For each pair of integers $(j, k)$ other than $(m, 1)$ define $r_{j k}$ to be $t_{j k}$ if $t_{j k} \neq 0$ and 1 if $t_{j k}=0$; set $r_{m 1}=1 / r_{11} r_{21} \cdots r_{m-1,1}$. Consider the matrices

$$
\begin{aligned}
D_{1} & =\operatorname{diag}\left(r_{11}, \cdots, r_{1, n(1)}\right) r_{21} r_{31} \cdots r_{m 1}, \\
D_{2} & =\operatorname{diag}\left(r_{21}, \cdots, r_{2, n(2)}\right) r_{31} r_{41} \cdots r_{m 1}, \\
\vdots & : \\
D_{m-1} & =\operatorname{diag}\left(r_{m-1,1}, \cdots, r_{m-1, n(m-1)}\right) r_{m 1}, \\
D_{m} & =\operatorname{diag}\left(r_{m 1}, \cdots, r_{m, n(m)}\right),
\end{aligned}
$$

and let $D=\operatorname{diag}\left(D_{1}, \cdots, D_{m}\right)$. Then $D^{-1} B D$ is similar over $F$ to $B$. Straightforward computation shows that the nonzero entries of $D^{-1} B D$ are all 1 except possibly for the position $(n(1)+\ldots$ $+n(m-1)+1,1)$; the entry at this position is $r_{11} r_{21} \cdots r_{m-1,1} t_{m 1}$ $= \pm \operatorname{det} A$. Hence $D^{-1} B D$ is real. This completes the proof of sufficiency for the condition that $\operatorname{det} A$ be real; the necessity is immediate.

TheOREM 3. Let $U$ be a complex unitary matrix. Then $\operatorname{det} U= \pm 1$ if and only if $U=J_{1} J_{2} J_{3} J_{4}$, where the $J_{i}$ are symmetries and $J_{1} J_{2}$ commutes with $J_{3} J_{4}$.

Proof. Assume det $U= \pm 1$. We can actually draw a stronger conclusion than mentioned above. Let $F$ be the smallest subfield of complex numbers containing the entries of $U$ together with its eigenvalues. We shall show that the desired matrices $J_{i}$ can be chosen over $F$.

Since $U$ is unitarily equivalent over $F$ to a diagonal matrix, we can assume that $U=\operatorname{diag}\left(\alpha_{1}, \cdots, \alpha_{n}\right)$. Define $n \times n$ unitary matrices $V$ and $W$ by

$$
\begin{aligned}
& V=\operatorname{diag}\left(\alpha_{1}, \bar{\alpha}_{1}, \alpha_{1} \alpha_{2} \alpha_{3}, \bar{\alpha}_{1} \bar{\alpha}_{2} \bar{\alpha}_{3}, \cdots, \alpha_{1} \alpha_{2} \cdots \alpha_{2 j+1},\right. \\
& \left.\bar{\alpha}_{1} \bar{\alpha}_{2} \cdots \bar{\alpha}_{2 j+1}, \cdots\right), \\
& W=\operatorname{diag}\left(1, \alpha_{1} \alpha_{2}, \bar{\alpha}_{1} \bar{\alpha}_{2}, \cdots, \alpha_{1} \alpha_{2} \cdots \alpha_{2 j}, \bar{\alpha}_{1} \bar{\alpha}_{2} \cdots \bar{\alpha}_{2 j}, \cdots\right) .
\end{aligned}
$$

If $n=2 k$, then all the complex eigenvalues of $V$ appear in conjugate pairs, so that it is the product of two symmetries over $F$; this is a special case of the result of [2], but can be verified directly by observing that

$$
\left(\begin{array}{ll}
\beta & 0 \\
0 & \bar{\beta}
\end{array}\right)=\left(\begin{array}{ll}
0 & \beta \\
\bar{\beta} & 0
\end{array}\right)\left(\begin{array}{ll}
0 & 1 \\
1 & 0
\end{array}\right) .
$$

Also, the last diagonal entry of $W$ is $\alpha_{1} \alpha_{2} \cdots \alpha_{2 k}=\operatorname{det} A$, which is \pm 1 by hypothesis; hence $W$ is also the product of two symmetries 
over $F$. If $n$ is odd, then the last diagonal entry of $V$ is $\operatorname{det} A$, and a similar argument proves the desired result.

REMARKS. (i) For $n>2$ there are $n \times n$ unitary matrices with real determinant which cannot be expressed as the product of three (or less) hermitian matrices: Let $\alpha=\exp (\pi i / n)$ and let $I$ denote the $n \times n$ identity. If $\alpha I=M_{1} M_{2} M_{3}$, where the $M_{j}$ are hermitian, then $\alpha M_{1}^{-1}$ is similar to its adjoint; hence $\alpha^{2} M_{1}^{-1}$ and $M_{1}^{-1}$ are similar and have the same spectrum. This is a contradiction since $M_{1}^{-1}$ is hermitian and $\alpha^{2}$ is nonreal. Every $2 \times 2$ unitary matrix with real determinant is the product of three symmetries.

(ii) If $U$ is a real unitary matrix, then it is the product of two real symmetries. This can be deduced from Theorem 1 as follows: $U=M_{1} M_{2}$ where $M_{i}$ is real hermitian. Every invertible real hermitian matrix is the product of a real positive-definite matrix and a real symmetry commuting with it. Write $M_{i}=H_{i} J_{i}$ accordingly, where $H_{i}$ is positive-definite and $J_{i}$ is a symmetry. Then $J_{1} U J_{2}=H_{1} H_{2}$ so that $H_{1} H_{2}$ is unitary; since the spectrum of $H_{1} H_{2}$ is necessarily real and positive, we have $H_{1} H_{2}=I$.

(iii) If a complex matrix has nonreal determinant, then it is not a finite or infinite product of hermitian matrices. The infinite product is of course interpreted as a limit of finite products in any norm on the linear space of matrices; det $X$ is a continuous function of $X$.

A complex matrix $A$ is the product of two hermitian matrices if and only if it is similar to a real matrix [1]; this occurs if and only if $A$ is similar to its adjoint [5]. If $A$ is unitary, then $A$ is the product of two symmetries if and only if $A$ is similar to $A^{*}$ [2]. Thus it would be interesting to characterize those complex matrices which are the product of three hermitian matrices and also those which are the product of three symmetries.

AnDED IN PROOF. C. S. Ballantine has pointed out that Theorem 1 was discovered by Frobenius in 1910.

\section{BIBLIOGRAPHY}

1. D. H. Carlson, On real eigenvalues of complex matrices, Pacific J. Math. 15 (1965), 1119-1129.

2. Chandler Davis, Separation of two linear subspaces, Acta. Sci. Math. (Szeged) 19 (1958), 172-187.

3. P. R. Halmos and S. Kakutani, Products of symmetries, Bull. Amer. Math. Soc. 64 (1958), 77-78.

4. Kenneth Hoffman and Ray Kunze, Linear algebra, Prentice-Hall, Englewood Cliffs, N. J., 1964, pp. 187-201.

5. Heydar Radjavi and James Williams, Products of self-adjoint operators, Michigan Math. J. (to appear).

UNIVERSITY OF TORONTO 